\title{
A NEW APPROACH OF BRAIN TUMOR SEGMENTATION USING FAST CONVERGENCE LEVEL SET
}

\author{
Virupakshappa $^{1}$ and Basavaraj Amarapur ${ }^{2}$ \\ ${ }^{1}$ Department of CSE, Appa IET, Kalaburagi, Karnataka, India \\ virupakshi.108@gmail.com \\ ${ }^{2}$ Department of E \& E E, P D A C E, Kalaburagi, Karnataka, India \\ bamarapurdyahoo.co.in
}

\begin{abstract}
Segmentation of region of interest has a critical task in image processing applications. The accuracy of Segmentation is based on processing methodology and limiting value used. In this paper, an enhanced approach of region segmentation using level set $(L S)$ method is proposed, which is achieved by using cross over point in the valley point as a new dynamic stopping criterion in the level set segmentation. The proposed method has been tested with developed database of MR Images. From the test results, it is found that proposed method improves the convergence performance such as complexity in terms of number of iterations, delay and resource overhead as compared to conventional level set based segmentation approach.
\end{abstract}

\section{KEYWORDS}

Fast convergence, dynamic stopping, Level set segmentation, valley pin point, cross over point.

\section{INTRODUCTION}

Image segmentation has remained an area of interest in image coding from its evolution. The accuracy of image coding and the processing efficiency of such systems are dependent on the processing algorithm used. The processing coefficient and the regions extracted are the major concern in deriving the resource overhead and its application in real time usage. Recently among various applications of medical image processing Magnetic Resonance Imaging (MRI) has gained high interest, due to new technology development in image capturing devices, which gives the digitized image details and the computation algorithm results in faster processing of such samples.

The automation of medical image processing and tumor processing has been focused, due to its higher criticality level. With the increase in new diseases, brain tumor is observed to be on higher rate. Various causes are responsible for the rise of such diseases extending from the common life style to genetical disorderness. However, many of the tumors are detected on the later stages which result in various disasters. It is easier in removal of a smaller tumor at the early stages, which minimizes the spreading risk as well health hazards.

DOI: $10.5121 /$ ijbes.2018.5102 
International Journal of Biomedical Engineering and Science (IJBES), Vol. 5, No. 1, January 2018

In the progress of automated process, computer based diagnosis has a higher degree of advantage, which are on a rapid development stage [1-5]. The development with the new technologies upcoming in visualization and modelling, has given a new paradigm for tumor detection. The critical format of the tumor structure has resulted in detection error and classification issues [6]. The available approach of visualization, has given the opportunity in building new approach of automation system based on learning approaches [9].

The tumor observed in the brain regions are broadly classified as 1) Benign Tumors, 2) Effected Tumor. Where in benign tumors are small tumor region spread to a small area region. This type of effects is limited to small region of brain and can be treated with surgery or radiation treatment. Whereas the effected type of tumor is observed to be highly effective, where tumor region spreads out in a random manner and an early detection could prevent it. In case of later stage detection this is highly effective.

A substantial methodological framework including new data analysis method was developed in [10] to meet the challenge of working with big data. The effective image coding determination for MRI and panoramic approach for surveillance for cancer detection in tumor image detection was outlined in [11]. Various stages of image processing were used for tumor detection using basic primary filtration, segmentation approach and filtration approach. The CAD system [12] has been developed with various additional input features such as EEG information and tumor symptoms. The EEG signal processing for tumor detection has an advantage of additional feature representation and given new advantage of tumor detection for lower grade tumor detection at early stage. An approach of tumor detection using Matlab is outlined in $[13,14]$. The approach defines the extraction and processing of MRI sample for tumor detection based on noise removal function, segmentation, and morphological operation. The approach of automated localization of the approach presented was outlined in this work. In [15] an artificial neural network approaches by Back propagation network and probabilistic neural network was presented. Osher and Sethian [16] presented a contour based coding called "Level set" (LS) method.

This method has the advantage to counter topological changes in the contours observed at the bounding regions of an image. Regularization of level set method brings an accuracy with the objective of faster segmentation, however, the initial seed selection and the stopping criterion are unbound in the conventional system. This leads to an over fitting problem, which intern result in slower segmentation process. The limiting criterion tends to manual errors and result in extra iterations or earlier stopping. This problem is addressed in this paper, and a novel approach of image segmentation for MRI samples is proposed. the defining approach, has an automated stopping criterion developed using the proposed approach called "valley point" (VP) approach, where the convergence is made dynamic by detecting crossover point in the valley region of level set variation.

The rest of the paper is presented in 6 sections. The conventional method of level set coding is outlined in section 2 . The proposed approach of modified level set coding is outlined in section 3 . Section 4 outlines the result obtained for the developed approach. The concluding remarks for the developed approach are made in section 5. 


\section{LEVEL SET SEGMENTATION}

Generally, the level set approach is a technique for tracking region and its bounding regions. The significance of level set method is the structural representation of the bounding region in an evolutionary manner. The curve evolution involves the surface and grid parameterization of the region. In level set approach, an initial zero level set is used as an initiation value to start the convergence. In the evolution is based on the emerging curvature region or the bounding region, which were taken as an interior shape region or zero at the outer bound region. The level set approach is defined as a closed curvature using defining function termed as level set function. In this approach, when the curve moves with as speed, the level set function need to satisfy,

$$
\frac{\partial \vartheta}{\partial t}=v|\nabla \vartheta|
$$

Here, I.I is defined as the Euclidean norm, and ' $t$ ' is time of observation. The level set function is defined as a partial differential equation, which follow the Hamilton-Jacobi equation, and solved using finite difference and Cartesian grid method. The bounded region is defined as an evolving of 2 dimensional data in a 3 dimensional space. The smoothing parameter for this function is given by, $(x, y, t)$, where $\vartheta(x, y, t)=0$, represents the closing boundness of the curve. For a given level set $\vartheta(x, y, t)=0$, set as a zero level set function, the whole region is divided into internal and external region of curve. The Signed Distance Function (SDF) is given by,

$$
\vartheta(x, y, t)=0=d
$$

Where ' $\mathrm{d}$ ' is the shortest distance between the points on the surface and the curve. In the evolution process, it is traced to satisfy the constraint,

$$
\vartheta(x, y, t)=0
$$

The movement formula for the curve evolution is given by,

$$
\vartheta_{t}+v|\nabla(\vartheta)|=0
$$

Here ' $v$ ' is the speed function, which is defined with the evolution of curve and the gradient of the image pixel. The speed function is defined as a function of the image information and the ideal value of zero at the target edge region. The curve evolution is dependent on the gradient of the image pixel in this case.

The basic approach in this method is to localize the region into two regions of required regions and the background. For a simple case I $(\mathrm{x}, \mathrm{y})$ has two regions which are denoted by the intensity values of $\mathrm{c} 1$ and $\mathrm{c} 2$. The curve of region 1 is denoted by $\mathrm{c}_{0}$ the fitting function defined by Mumford-shah [15] model is given by,

$$
\begin{gathered}
F\left(c_{1}, c_{2}, C\right)=\mu(\text { length }(C)+v(\operatorname{area}(\text { inside }(C)) \\
=\alpha_{1} \int_{\text {inside }(C)}\left|I(x, y)-c_{1}\right|^{2} d x d y+\alpha_{2} \int_{\text {outside }(C)}\left|I(x, y)-c_{2}\right|^{2} d x d y
\end{gathered}
$$


International Journal of Biomedical Engineering and Science (IJBES), Vol. 5, No. 1, January 2018

Where ' $\mathrm{C}$ ' is the evolving curve, and ' $c_{1}{ }^{\prime},{ }^{\prime} c_{2}$ ' are the constant values. The length(c) is the length of the closed border $\mathrm{c}$ and area inside is the area under the bounding curve. $\alpha_{1}, \alpha_{2}$ are the step constant parameters. The two constant values are defined by the user observation and the limiting issue is then lead to either over fitting or constraint iteration. This process leads to slower curve evolution or low accurate curves. These limitations bound the efficiency of level set approach in critical applications, such as MRI image segmentation, where faster and accurate image segmentation is a prime need. To overcome the issue of stopping criterion, in this paper, a fast convergence coding approach is suggested. This approach has the significance of accurate and exact stopping of curve evolution, improving segmentation efficiency.

\section{Proposed Valley Peak fast Convergence Coding}

For the region segmentation of a tumor in a MRI sample, level set coding is defined as a set of points. These set of points are evolved towards an edge region, where the edge region and forced to move towards the bounding region governed by the gradient of the evolution characteristics. The level set function is given by equation (6) defines two evolution regions, where the initial region is defined by the evolution of contour region having pixel values greater than a limiting value of $c_{1}$. This approach of evolution, i.e. contour which is based on the limiting condition is called the soft thresholding approach. The second factor tries to converge towards the outer boundary region in the image. Here the region movement is based on the gradient map and the convergence of region is limited by the minimization criterion of $\vartheta(x, y, t)=0$. Here the evolution is a derived as a set of contour and as observed based on image intensities. It is observed that, the region convergence depends on the iterations given or the minimization criterion set. The convergence is achieved once the curve tends to the minimization of $\vartheta(x, y, t)$. However, the bounding condition of the curve evolution is limited with user defined values, which tends to the constraint of over computation or under computation issue. These issues leads to delay or lower accuracy in segmentation process.

As the curve evolution is a function of image gradient $|\nabla \vartheta|$, the bounding condition cannot be limited for segmentation. The gradient variation is observed to be dynamic for each sample and hence dynamic limiting values are appropriate. This leads to the development of automated bounding consist in level set coding for faster and accurate converges. To achieve this objective an approach called "Valley peak" estimation is proposed. the approach is based on the characteristic analysis of the level set variation.

In the evolution of level set function, it is observed that, once the convergence criterion met, i.e. The cost function is achieving the curve evolution is to be terminated.

The cost function is defined as

$$
C f=\min \left(\left|I(x, y)-c_{1}\right|^{2}\right), \rightarrow(\vartheta(x, y, t)=0
$$

The minimum difference in the difference in closed region of curve evolved tending to the curve bounding condition of $(\vartheta(x, y, t)=0$

With this objective the level set function is defined with a new bounding condition defined as,

$$
\alpha_{1} \int_{\text {inside }(C)}^{z<(z-1)}\left|I(x, y)-c_{1}\right|^{2} d x d y+\alpha_{2} \int_{\text {outside }(C)}^{z<(z-1)}\left|I(x, y)-c_{2}\right|^{2} d x d y
$$


International Journal of Biomedical Engineering and Science (IJBES), Vol. 5, No. 1, January 2018

The level set function is bounded here with the condition of a zero cross value $z$ defined by

$$
Z=\vartheta(t)-\vartheta(t+1)
$$

Which is defined as the variation in the two successive observation at ' $t$ ' and ' $t+1$ ' of generated level set function defining the curve evolution.

The termination condition is defined as,

$$
\begin{aligned}
& \text { if } Z<0, S=1 \\
& \text { else } \\
& S=0
\end{aligned}
$$

The terminating status $\mathrm{S}$ is set high to stop the curve evolution condition once the zero cross value is obtained. This variation is illustrated in figure 1 .

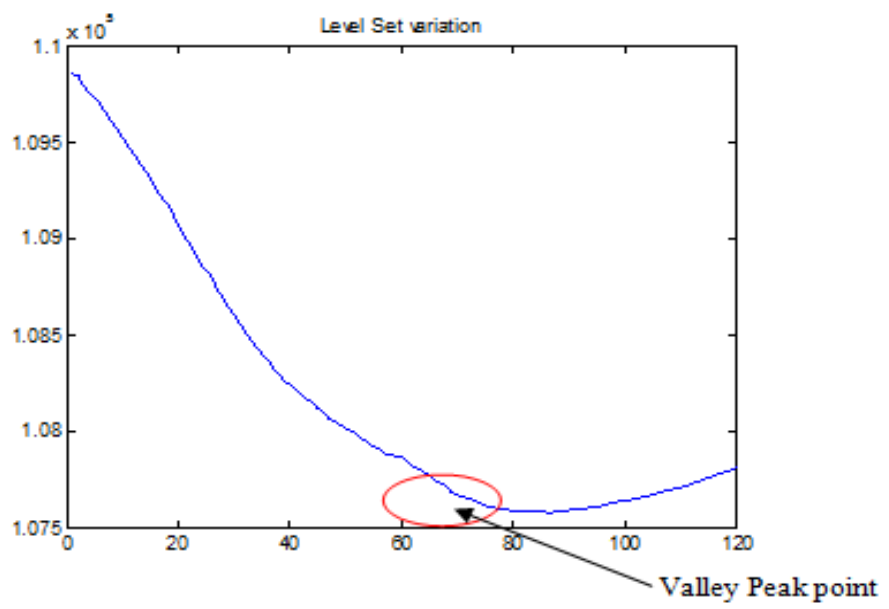

Figure 1: Valley peak point in a Level Set evolution

This characteristic analysis illustrates a sharp variation in the level set evolution, where it is observed that the levels set value tends to lower until the bounding curve regions are detected. There in after the curve increases due to evolution in region c2, which is observed to have the higher gradient variation with curve evolution process. The valley peak point in this evolution represent the barrier of translating the process from $\mathrm{C} 1$ to $\mathrm{C} 2$ region, hence a termination can be made at this point.

\section{EXPERIMENTAL RESULTS}

The proposed system has been tested using different MR images of brain tumor, which were collected from Database as well as hospital. These images are in larger size of 1064 X 1064 resolution. They are resized using scrapping. The final scrapped images of size of $250 \times 250$ resolution with gray colour intensity is as shown in figure 2, which consists of tumor region as well as suspicious non tumor region. 
International Journal of Biomedical Engineering and Science (IJBES), Vol. 5, No. 1, January 2018

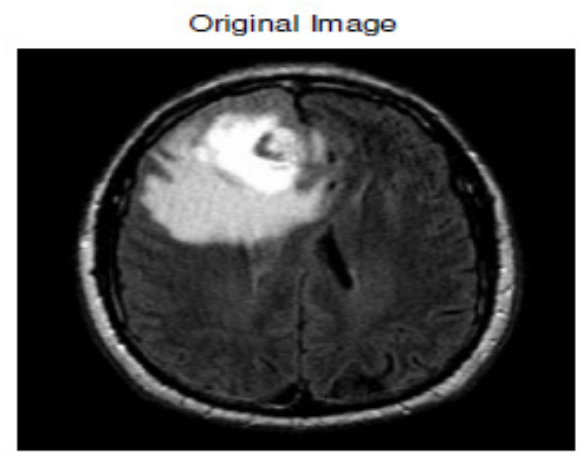

Figure 2: Original MRI Test sample

In order to segment the tumor region, the proposed algorithm has been applied which results the iteration of level set starts from the seed point. The selection of the seed point was carried out automatically using a highest pixel intensity in the image. The evaluation of the level set has been carried out with the constraint and tending to optimize the cost function given by equation (7). The evaluation terminates at valley peak point i.e. zero cross value is obtained. The segmented region of interest is represented by red curve as shown in figure 3 .

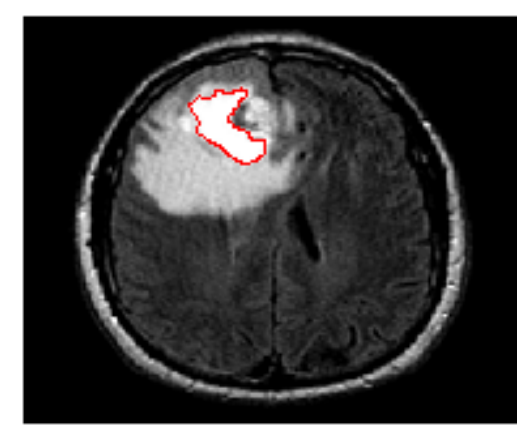

Figure 3: Bounding Region Marked for the tumorous region

Then the final segmented tumor region from the MR image is as shown in figure 4.

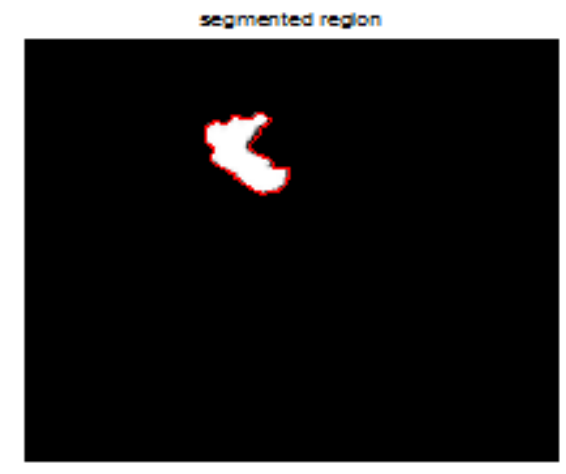

Figure 4: Segmented Tumor Region from the MRI sample 
International Journal of Biomedical Engineering and Science (IJBES), Vol. 5, No. 1, January 2018

The characteristic analysis of proposed method has been carried out with the following observations and computation issues are discussed as compared to DRLSE method.

The area variation graph is drawn as shown in figure 5, which is plotted as area of segmented image versus number of iterations. Using proposed algorithm, the variation of area is between 80 and 82 iterations as compared to DRLSE method is approximately 120 iterations.

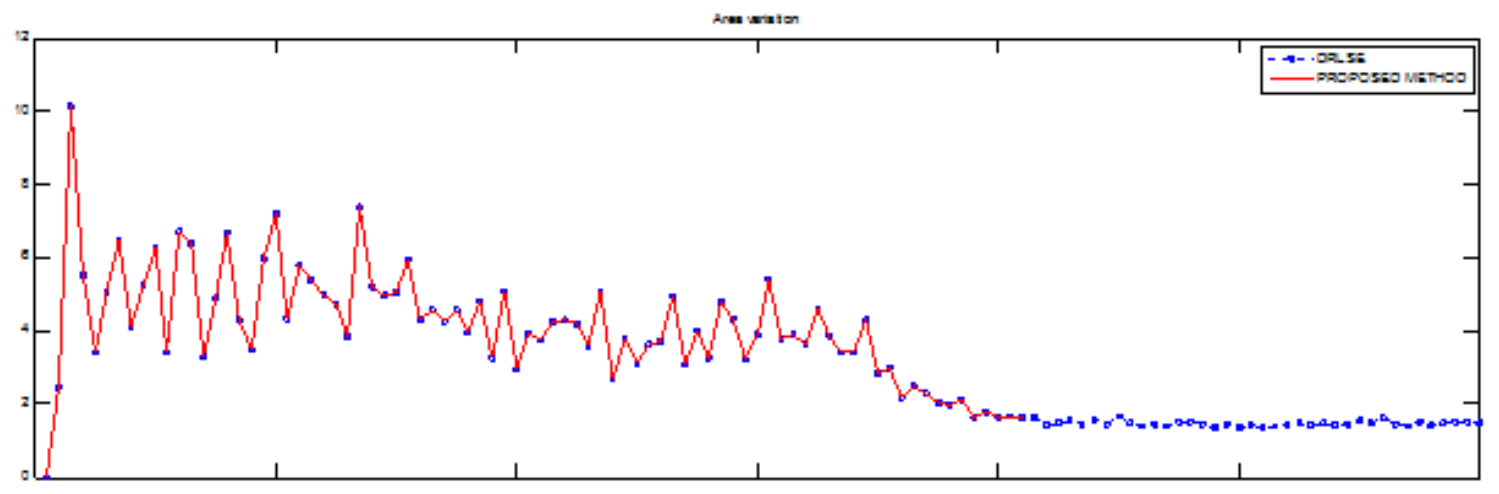

Figure 5. Area Variation.

Similarly, the edge variation analysis is also carried out and is as shown in the figure 6.

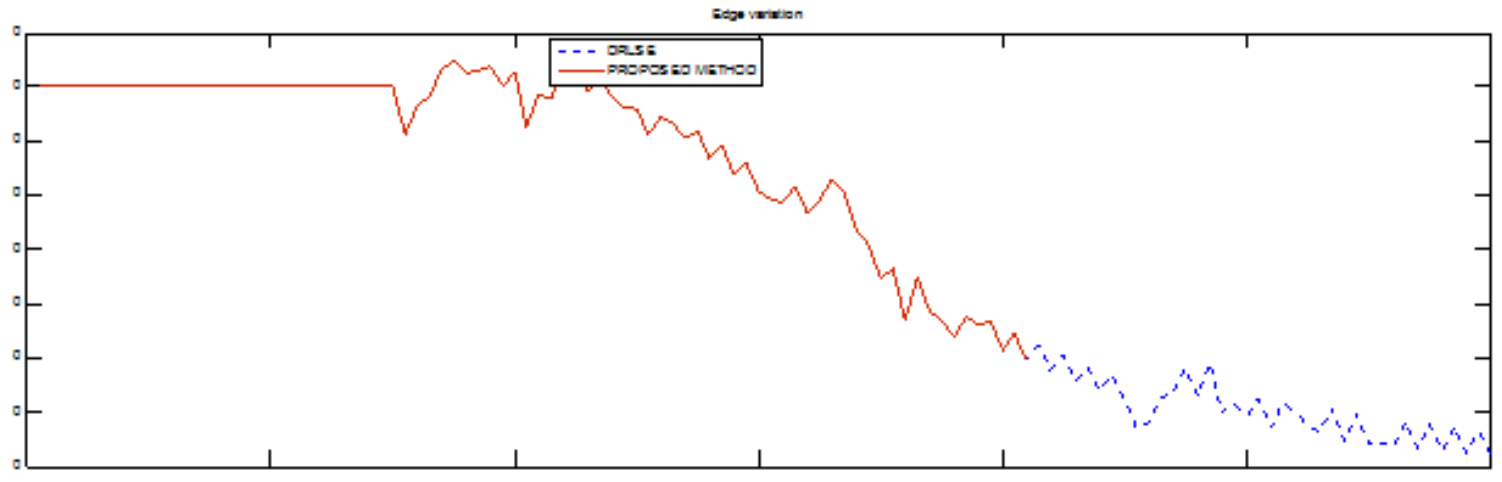

Figure 6. Edge Variation

From the graph, the edge variation is significant till $72^{\text {nd }}$ iteration for the proposed approach and 120 plus iteration for the DRLSE method.

Finally, the level set characteristic is illustrated in figure 7 . The variation observed clearly reveals a zero crossing detection at 80 iterations. The computation made after 80 is the over computation which results in the same tumor region but with additional computation effort. 
International Journal of Biomedical Engineering and Science (IJBES), Vol. 5, No. 1, January 2018

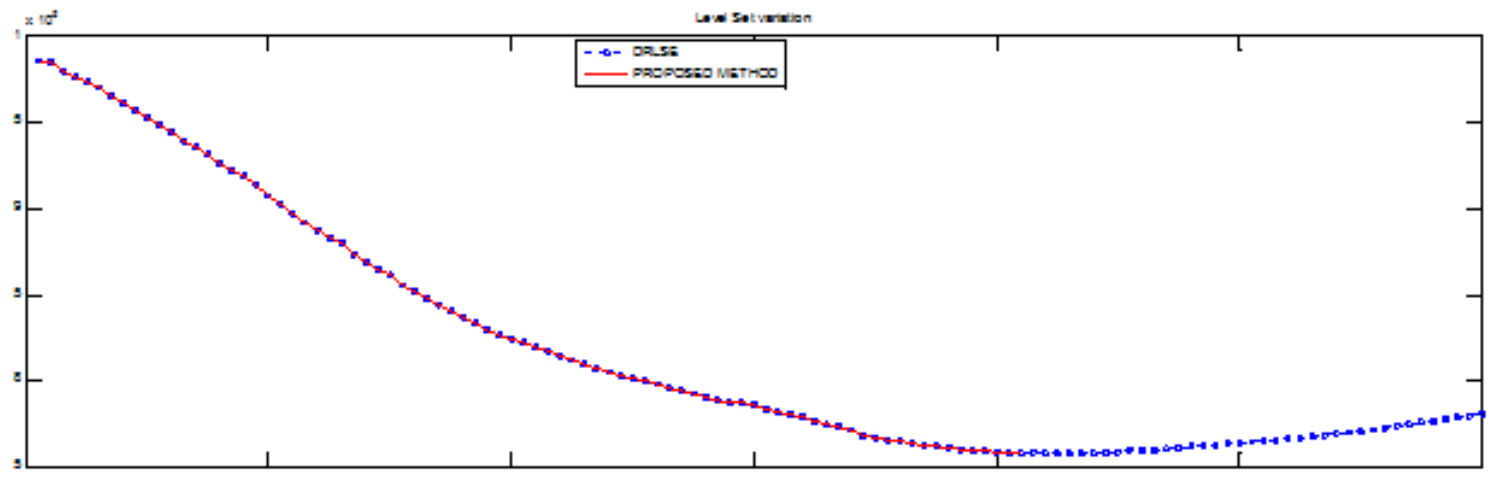

Figure 7. Level set Variation

A similar test is carried out for the validation of different tumor region. The obtained restful observation for a test sample is presented below.

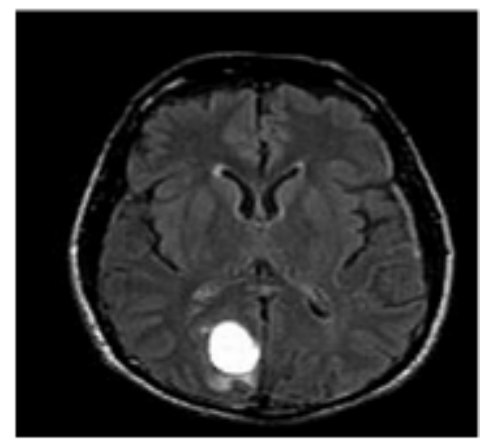

Figure 8: original Test sample

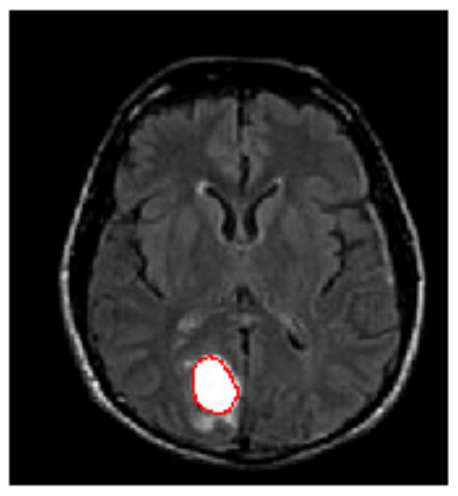

Figure 9: detected tumor region 
International Journal of Biomedical Engineering and Science (IJBES), Vol. 5, No. 1, January 2018

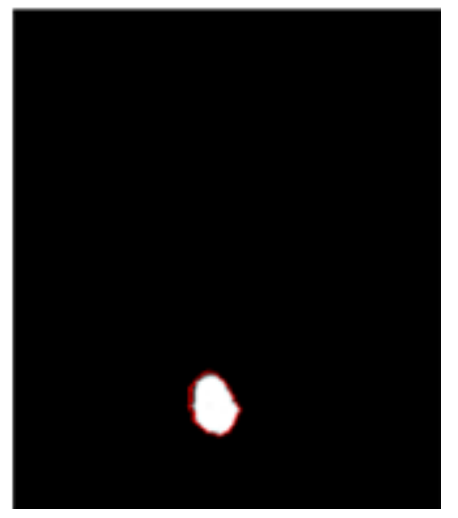

Figure 10: segmented tumor region

Finally, the comparison between proposed method and conventional DRLSE method has been carried out in terms of convergence time in seconds. The results obtained are tabulated in the table 1 .

Table 1: Convergence Time (Sec) observed for the developed approach

\begin{tabular}{|c|c|c|}
\hline Sample & De-Regularized Approach [1] & VP-Regularized Approach \\
\hline & & 32.2 \\
\hline & 44.3 & \\
\hline & & \\
\hline & 56.7 & \\
\hline & & \\
\hline & & \\
\hline
\end{tabular}

From the results it shows that the computation times for sample 1, sample 2 and sample 3 using proposed method are 32.2, 27.3 and 37.4 respectively as compared to DRLSE method i.e. 44.3, 56.7 and 49.3 respectively. From the comparison, it shows that the proposed method's computation time is less than the DRLSE method. The same is shown in figure 11 using convergence plot. 
International Journal of Biomedical Engineering and Science (IJBES), Vol. 5, No. 1, January 2018

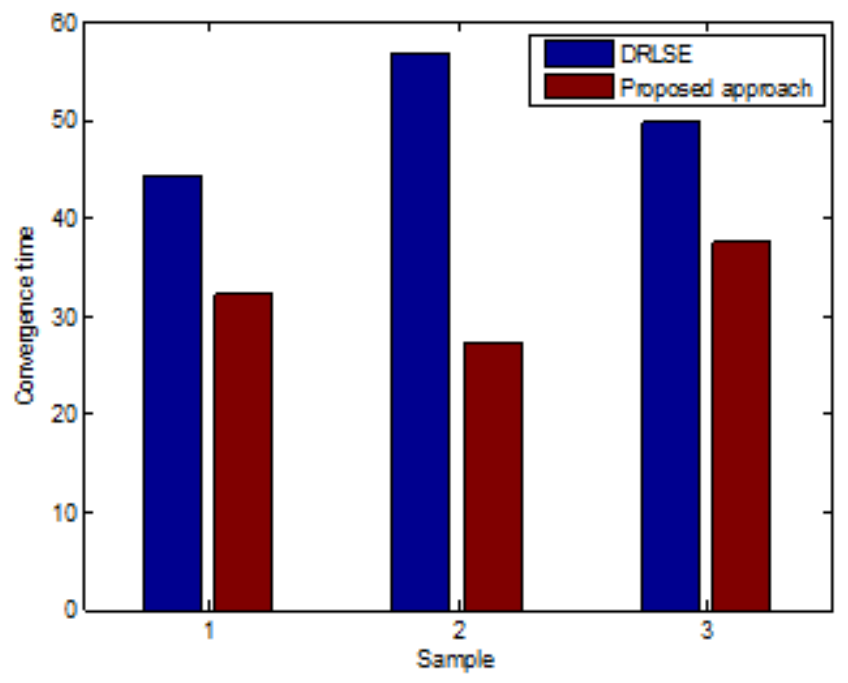

Figure 11: convergence plot

\section{CONCLUSIONS}

An approach to image segmentation based on level set approach is developed for an MRI tumor image detection. level set coding where the valley coding is used for region marking as stopping criterion. The convergence time for the region detection and segmentation is comparatively faster for the proposed approach as compared to the conventional distance-regularizing approach. The curve evolution approach in this case is observed to be faster converged due to appropriate detection of region variation in level set obtained for MRI sample. The comparison in the detected approach illustrate the accuracy of region localization with faster convergence.

\section{REFERENCES}

[1] Ma, J., Lu, L.. Hierarchical segmentation and identification of thoracic vertebra using learning-based edge detection and coarse-to-fine deformable model. Computer Vision and Image Understanding 2013;117(9):1072-1083.

[2] Klinder, T., Ostermann, J., Ehm, M., Franz, A., Kneser, R., Lorenz, C.. Automated model-based vertebra detection, identification, and segmentation in CT images. Medical Image Analysis 2009;13(3):471-482.

[3] Rak, M., T"'onnies, K.D.. On computerized methods for spine analysis in MRI: a systematic review. International Journal of Computer Assisted Radiology and Surgery 2016;:1-21.

[4] S.-H., H., Chu, Y.H., Lai, S.H., Novak, C.L.. Learning-based vertebra detection and iterative normalized-cut segmentation for spinal MRI. IEEE Transactions on Medical Imaging 2009;28(10):1595-1605.

[5] Hoad, C.L., Martel, A.L.. Segmentation of MR images for computer-assisted surgery of the lumbar spine. Physics in Medicine and Biology 2002;47(19):3503.

[6] Kass, M., Witkin, A., Terzopoulos, D.. Snakes: Active contour models. International Journal of Computer Vision 1988;1(4):321-331. 
International Journal of Biomedical Engineering and Science (IJBES), Vol. 5, No. 1, January 2018

[7] Caselles, V., Kimmel, R., Sapiro, G.. Geodesic active contours. International Journal of Computer Vision 1997;22(1):61-79. 8. Cootes, T.F., Taylor, C.J., Cooper, D.H., Graham, J.. Active shape models-their training and application. Computer Vision and Image Understanding 1995;61(1):38-59.

[8] Davatzikos, C., Liu, D., Shen, D., Herskovits, E.H.. Spatial normalization of spine MR images for statistical correlation of lesions with clinical symptoms 1. Radiology 2002;224(3):919-926.

[9] Stern, D., Likar, B., Pernu`s, F., Vrtovec, T.. Parametric modelling and segmentation of vertebral bodies in 3d CT and MR spine images. Physics in Medicine and Biology 2011;56(23):7505.

[10] Neubert, A., Fripp, J., Shen, K., Salvado, O., Schwarz, R., Lauer, L., et al. Automated 3d segmentation of vertebral bodies and intervertebral discs from MRI. In: Proceedings of Digital Image Computing Techniques and Applications (DICTA) 2011. 2011, p. 19-24.

[11] Neubert, A., Fripp, J., Engstrom, C., Schwarz, R., Lauer, L., Salvado, O., et al. Automated detection, $3 \mathrm{~d}$ segmentation and analysis of high resolution spine MR images using statistical shape models. Physics in Medicine and Biology 2012;57(24):8357.

[12] Ayed, I.B., Punithakumar, K., Minhas, R., Joshi, R., Garvin, G.J.. Vertebral body segmentation in MRI via convex relaxation and distribution matching. In: Proceedings of Medical Image Computing and Computer-Assisted Intervention (MICCAI) 2012. 2012, p. 520- 527.

[13] Zuki'c, D., Vlas'ak, A., Egger, J., Ho־r'snek, D., Nimsky, C., Kolb, A.. Robust detection and segmentation for diagnosis of vertebral diseases using routine MR images. Computer Graphics Forum 2014;33(6):190-204.

[14] Zhang, Y., Matuszewski, B.J., Shark, L.K., Moore, C.J.. Medical image segmentation using new hybrid level-set method. In: Proceedings of BioMedical Visualization (MEDIVIS) 2008. 2008, p. 7176.

[15] Sethian, J. A. Level set methods and fast marching methods: evolving interfaces in computationalgeometry, fluid mechanics, computer vision, andmaterials science. Cambridge University Press, 1999.

[16] Osher S, Sethian J A. Fronts propagating with curvature-dependent speed: algorithms based on Hamilton-Jacobi formulations[J]. Comput. Phys, 1988; 79(1): 12-49.

\section{AUTHORS}

Virupakshappa completed B.E in Computer Science \& Engineering from Khaja Banda Nawaz College of Engineering Gulbarga in the year 2008 and Masters in Computer Science \& Engineering from Poojya Doddappa Appa College of Engineering Gulbarga in the year 2011. Currently he is working as Associate Professor in Department of CSE. Appa IET, Kalaburagi, Karnataka, India. He is pursuing his Ph.D. from VTU Belgaum. His area of interest is Biomedical Image Processing and Pattern Recognition.

Dr. Basavaraj Amarapur completed B.E. Electrical Engineering from H. K. E. Society's Engineering College Gulbarga in the year 1985 and Masters in computer science \& Engineering from University of Roorkee in the year 1991. He has completed $\mathrm{Ph}$. D. from VTU Belgaum. Currently he is working as Professor in the department of Electrical and Electronics Engineering, Poojya Doddappa Appa college of Engineering Kalaburagi, Karnataka, India. His research area of interest is Biomedical Image Processing, computer vision and Pattern Recognition.

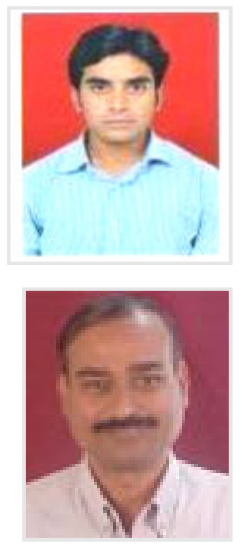

